\title{
Puff Models for Simulation of Fugitive Hazardous Emissions in Atmosphere
}

\author{
Ledina Lentz Pereira1, Camila Pinto da Costa ${ }^{2}$, Marco Tullio Vilhena ${ }^{3}$, Tiziano Tirabassi ${ }^{4}$ \\ ${ }^{1}$ University of the South End of Santa Catarina (UNESC), Criciúma, Brasil; ${ }^{2}$ Federal University of Pelotas (UFPel), Pelotas, Brazil; \\ ${ }^{3}$ Federal University of Rio Grande do Sul (UFRGS), Porto Alegre, Brazil; ${ }^{4}$ Institute of Atmospheric Sciences and Climate (ISAC), \\ National Research Council (CNR), Bologna, Italy. \\ Email:t.tirabassi@isac.cnr.it
}

Received November $10^{\text {th }}, 2010$; revised December $20^{\text {th }}, 2010$; accepted February $6^{\text {th }}, 2011$.

\begin{abstract}
A puff model for the dispersion of material from fugitive hazardous emissions is presented. For vertical diffusion the model is based on general techniques for solving time dependent advection-diffusion equation: the ADMM (Advection Diffusion Multilayer Method) and GILTT (Generalized Integral Laplace Transform Technique) techniques. Both approaches accept wind and eddy diffusion coefficients with any restriction in their height functions. Comparisons between values predicted by the models against experimental ground-level concentrations (from Copenhagen data set) are shown. The preliminary results confirm the applicability of the approaches proposed and are promising for future work.
\end{abstract}

Keywords: Hazardous Emissions, Advection-Diffusion Equation, Analytical Solutions, Air Pollution Modeling, Puff Models

\section{Introduction}

The study on hazardous material dispersion shows that the diffusion in the atmosphere depends strongly on the meteorological scenario and boundary layer evolution. Traditionally, the advection-diffusion equation has been largely applied in operational atmospheric dispersion models to predict the mean concentration of contaminants in the Atmospheric Boundary Layer (ABL). In principle, from this equation it is possible to obtain a theoretical model of dispersion from a point source given appropriate boundary and initial conditions plus knowledge of the mean wind velocity and turbulent fluxes.

Much of the turbulent researches are related to the specification of turbulent fluxes in order to allow the solution of the averaged advection-diffusion equation: this procedure, sometimes, is called as the closure of the turbulent diffusion problem. The main scheme for closing the equation has to take into account the relationship between concentration turbulent fluxes and the gradients of the mean concentration by exchange coefficients.

A broad class of numerical solutions to the advection-diffusion equation can be encountered in the literature. Nevertheless, the search of analytical solutions for this equation has several advantages. Indeed, in the ana- lytical solution all the influencing parameters are explicitly expressed in a mathematical closed form and conesquently sensitivity analysis over model parameters may be easily performed and, more important in order to manage alarms for fugitive hazardous emissions, air pollution model based on analytical formula run fast.

In fact, in order to model fugitive emissions, fast evaluation and relative decisions are necessary. So, fast codes are preferred and is better if they run in personal computer and can evaluate air pollution concentrations although some meteorological measures are present. Moreover, in the case of fugitive hazardous emissions, time dependent models are necessary.

Puff models are a practical approach to describe the dispersion from a time-dependent emission point source in an inhomogeneous and non-stationary ABL. Most of the operative puff models are based on the Gaussian approach (the shape of the puffs is Gaussian). Gaussian models are theoretically based upon an exact, but not realistic solution of the equation of transport and diffusion in the atmosphere, in cases where both wind and turbulent diffusion coefficients are constant with height. The solution is forced to represent real situations by means of empirical parameters, referred to as "sigma". The input parameters of the Gaussian plume model are 
often related to simple turbulence typing schemes or stability classes. The problem with such stability classes is that each covers a broad range of stability conditions; they are also very site specific and based towards neutral stability when unstable or convective conditions actually exist. Moreover, the shapes of puffs are not symmetric in vertical, while the Gaussian distribution is symmetric.

In fact, a distorting effect of the variation with height of the mean wind, both in speed and direction, is often evident in the development of puffs or plumes smoke. The effect is most evident in stably stratified conditions. In fact wind shear creates variance in the direction of the wind, vertical diffusion destroys this variance and tries to re-establish a non skewed distribution. The interaction between vertical mixing and velocity shear is continuously effective.

Conversely, Gaussian models are fast, simple, do not require complex meteorological input. For these reasons they are still widely employed for regulatory applications by environmental agencies all over the world. Nonetheless, because of their well known intrinsic limits, the reliability of a Gaussian model strongly depends on the way the dispersion parameters are determined on the basis of the turbulence structure of the ABL and the model's ability to reproduce experimental diffusion data. However, non-Gaussian puffs have been proposed in the literature [1-4].

In this paper we present two puff models where horizontal dispersion is Gaussian, but the vertical puff shape is non-Gaussian and it is evaluated by two different techniques that allow to obtain an analytical solution of the one-dimensional advection-diffusion equation: the GILTT (Generalized Integral Laplace Transform Technique) $[5,6]$ and ADMM (Advection Diffusion Multilayer Method) $[7,8]$ techniques.

The first one is a well-known hybrid method that had solved a wide class of direct and inverse problems mainly in the area of Heat Transfer and Fluid Mechanics and the solution is given in series form.

The second one is an analytical solution based on a discretization of the Atmospheric Boundary Layer (ABL) in sub-layers where the advection-diffusion equation is solved by the Laplace transform technique. The solution is given in integral form.

The models accept general profiles for eddy diffusivity coefficients, as well as the theoretical profiles proposed in the scientific literature, such as the vertical profiles of eddy diffusion coefficients predicted by the Similarity Theory.

\section{Puff Models}

Puff models were introduced to simulate the behaviour of pollutants in inhomogeneous and non-stationary mete- orological and emission conditions $[9,10]$. The emission is discretized in a temporal succession of puffs, each of which shifts into the area of calculus thanks to wind field.

Puff models assume that each emission of pollutants in a time interval $\Delta t$ releases into the atmosphere a mass of pollutants $\Delta M=Q \Delta t$, where $Q$ is the emission rate, which is variable in time. Each puff contains the mass $\Delta M$ and its centre of mass is transported by the wind, which may vary in space and time. Continuously emitting sources can be represented by the superposition of a series of the above clouds.

The puffs considered here are emitted in time intervals $\Delta t_{1}$ and the calculation of the concentration of pollutants of each one is made in a time intervals $\Delta t_{2}$. Each puff is carried in accordance with the trajectory from its centre, which is determined for velocity vector of the local wind, while it is enlarged in the time by means of the dispersion coefficients. In particular, in our model, the vertical diffusion of the material carried in accordance with the trajectory of puff is non-Gaussian and it is described by general solutions of the following equation:

$$
\frac{\partial c(z, t)}{\partial t}=\frac{\partial}{\partial z}\left(K(z) \frac{\partial c(z, t)}{\partial z}\right)+S
$$

for $0<z<h$ and the time $t>0$, subject to the boundary condition

$$
K_{z} \frac{\partial c}{\partial z}=0 \quad \text { for } \quad z=0 ; h
$$

and the initial condition: $c(z, 0)=0$. Here, $C(z, t)$ denotes the average crosswind integrated concentration, $h$ the ABL height, $K_{z}$ the eddy diffusivity coefficient, $S$ the instantaneous point source expressed like:

$$
S=Q \delta\left(t-t_{0}\right) \delta\left(z-H_{s}\right)
$$

In addition, $\delta$ represents the delta function, $z$ is the vertical variable, $t_{0}$ initial time, $H_{s}$ the source height.

In the sequel we solve problem (1) by the GILTT and ADMM approach.

The main idea of the GILTT approach consist in the expansion of the pollutant concentration in a truncated series of eigenfunctions. Replacing this expansion in the advection diffusion equation and taking moments, we come out with a first order linear matrix equation, which is then solved by the Laplace Transform technique. This procedure leads to a solution expressed in series formulation for more details see the work [6].

The main idea of the ADMM approach relies on the discretization of the PBL in a multilayer system, where in each layer the eddy diffusivity and wind profile assume averaged values. The resulting advection-diffusion equation in each layer is then solved by the Laplace 
Transform technique. For more details about this methodology see the work [11].

To construct a three-dimensional solution, we assume the Gaussian model in $\mathrm{x}$ and $\mathrm{y}$ directions for the pollutant concentration, therefore Gaussian solution reads like:

$$
\begin{aligned}
& \Psi_{\text {puff }}(x, y, t) \\
& =\frac{1}{2 \pi \sigma_{y} \sigma_{x}} \exp \left[-\frac{1}{2}\left(\frac{x-x_{0}}{\sigma_{x}}\right)^{2}\right] \\
& \times \exp \left[-\frac{1}{2}\left(\frac{y-y_{0}}{\sigma_{y}}\right)^{2}\right]
\end{aligned}
$$

where $x_{0}=u \Delta t, y_{0}=v \Delta t$ and $x_{0}, y_{0}$ are the centroid coordinates $(u$ and $v$ are the components of horizontal velocity of the average wind) and $\sigma_{x}, \sigma_{y}$ the lateral dispersion parameters [12] defined as:

$$
\sigma_{\alpha}=\sigma_{u_{a}} t f_{\alpha}\left(t / T_{\alpha}\right)
$$

where $\alpha$ means $x$ and $y, f_{\alpha}$ is the non-dimensional function of the travel time $t / T_{\alpha}$ :

$$
f_{\alpha}=\frac{1}{1+\sqrt{t / 2 T_{\alpha}}}
$$

where $T_{\alpha}$ is the Lagrangean scale of time for the horizontal $(x$ and $y)$ dispersion and $\sigma_{u_{\alpha}}$ is the standard deviation of the longitudinal and lateral components of the wind speed defined as:

$$
\left(\sigma_{u_{a}} / u_{*}\right)^{2}=0.35\left(-\frac{H_{S}}{k L}\right)^{2 / 3}+\left(2-h / H_{s}\right),
$$

where $u_{*}$ is friction velocity, $k$ is the Von Kárman constant $(k=0.4), \mathrm{h}$ is the height of the $\mathrm{ABL}$ and $\mathrm{L}$ is the Monin-Obukhov length.

\subsection{The Solution of the One-Dimensional Transient Problem by the GILTT Method}

In order to solve the Equation (1), we rewritten like:

$$
\frac{\partial c}{\partial t}=K^{\prime}(z) \frac{\partial c}{\partial z}+K(z) \frac{\partial^{2} c}{\partial z^{2}}+Q \delta\left(t-t_{0}\right) \delta\left(z-H_{S}\right)
$$

According the works [6] the solution of problem (1) is written like:

$$
c(z, t)=\sum_{i=0}^{\infty} \frac{\overline{A_{i}(t)} Z_{i}(z)}{N_{i}^{1 / 2}}
$$

where $Z_{i}(z)=\cos \left(\lambda_{i} z\right)$ and $\lambda_{i}=i \pi / h$ are respecttively the eigenfunctions and eigenvalues, and $\overline{A_{i}(t)}$ is the solution of the transformed problem, and $N_{i}$ is expressed by $N_{i}=\int_{v} Z_{i}^{2}(z) \mathrm{d} v$.
Replacing the Equation (9) in Equation (8) and taking moments. Thus, after we get the following first order linear matrix equation

$$
E Y^{\prime}(t)-B Y(t)=Q \delta\left(t-t_{0}\right) F
$$

For $t>0$, with $\mathrm{E}, \mathrm{Y}, \mathrm{B}$ and $\mathrm{F}$ defined as:

$$
\begin{aligned}
& E=\left[e_{i j}\right] \text { with } e_{i j}=\int_{0}^{a} \frac{Z_{i}(z) Z_{j}(z)}{N_{i}^{1 / 2} N_{j}^{1 / 2}} \mathrm{~d} z, \\
& Y(t)=\left[\overline{A_{j}(t)}\right], \\
& F=\left[f_{i j}\right] \quad \text { where } \quad f_{i}=\frac{Z_{i}\left(H_{s}\right)}{N_{i}^{1 / 2}},
\end{aligned}
$$

and the $B$ matrix is expressed by: $B=\left[b_{i j}\right]$, with

$$
\begin{aligned}
b_{i j}= & \frac{1}{N_{i}^{1 / 2} N_{j}^{1 / 2}} \\
& \times\left[\int_{0}^{a} k^{\prime}(z) Z_{i}(z) Z_{j}^{\prime}(z) \mathrm{d} z-\lambda_{j}^{2} \int_{0}^{a} k(z) Z_{i}(z) Z_{j}(z) \mathrm{d} z\right]
\end{aligned}
$$

For the initial condition, the procedure is analogous and after the substitutions due and integrations we have:

$$
\overline{A_{j}(0)}=0
$$

In this work the transformed problem represented by Equation (10) is solved by the Laplace Transform technique and diagonalization [13].

Thus, the final solution is given by

$$
Y(t)=T_{a} G_{D}(t) \xi
$$

where $\xi$ is the integration constant vector, $G_{D}(t)$ is the diagonal matrix witch elements are $e^{-d_{i}\left(t-t_{0}\right)}, T_{a}$ is the eigenfunction matrix and $d_{i}$ are the eigenvalues of the matrix $B$. [14].

The state-of-art the GILTT method can be found in

\subsection{The Solution of the One-Dimensional Problem Transient by the ADMM Method}

To solve the advection-diffusion Equation (1), for inhomogeneous turbulence by the ADMM method, we must take into account the dependence on the eddy diffusivities, on the height variable (variable $z$ ). To reach this goal we discretize the height $h$ of the ABL into $\mathrm{N}$ sub-intervals in such manner that inside each sub-region $K(z)$ assume respectively the following average values:

$$
K_{n}=\frac{1}{z_{n+1}-z_{n}} \int_{z_{n}}^{z_{n+1}} K_{z}(z) \mathrm{d} z
$$


Obviously, the greater the number of layers $(N)$, the more accurate the concentration pattern calculated, although the relative code running time is consequently greater. Moreover, the layers in which the ABL is divided can be not constant in thickness. A more detailed description is required of wind and diffusion coefficients in proximity to the ground, where their gradients are high and more strongly influence pollutant dispersion. Therefore layers close to the terrestrial surface can be assigned a smaller thickness than those located higher up. With the division of the domain into $\mathrm{N}$ sub-intervals, the solution of the Equation (1) is reduced to the solution of $N$ problems of this type:

$$
\frac{\partial c_{n}}{\partial t}=K_{n} \frac{\partial^{2} c_{n}}{\partial z^{2}}+Q \delta(z-H s) \delta\left(t-t_{0}\right)
$$

for $n=1, \cdots, N-1$, where, $c_{n}$ represents the concentration in the nth generic sub-layer. The boundary and initial conditions are given by:

$$
K_{n} \frac{\partial c_{n}}{\partial z}=0, \text { for } z=0, h
$$

and

$$
c_{n}(z, t)=0, \text { for } t=0
$$

The equations represented by the Equation (18) are joined by the continuity conditions for the concentration and flux at the interfaces. Namely:

$$
\begin{array}{ll}
c_{n}=c_{n+1} & n=1,2, \cdots,(N-1), \\
K_{n} \frac{\partial c_{n}}{\partial z}=K_{n+1} \frac{\partial c_{n+1}}{\partial z} & n=1,2, \cdots,(N-1) .
\end{array}
$$

We apply the Laplace Transform technique in time variable in Equation (18). We determine the solution of the resulting equation, in a easy manner, using the well know results of second order linear differential equations with constant coefficients. Indeed, it turns out that by this procedure, the pollutant concentration in each sub-layer reads like:

$$
\begin{aligned}
c_{n}(z, s) & =A_{n} e^{-R_{n} z}+B_{n} e^{R_{n} z} \\
& +\frac{Q}{2 R_{n} K_{n}}\left(e^{-\left(R_{n}(z-H)+t_{0} s\right)}-e^{R_{n}(z-H)-t_{0} s}\right) .
\end{aligned}
$$

for $n=1, \cdots, N-1$, whereas $s$ denotes the Laplace transformed variable. We are now in position to construct the global solution for the puff emission. For such, ac- cording the Green function theory for particular solution, we write down the solution for a single puff like:

$$
\begin{aligned}
c_{n}(z, t)= & \frac{1}{2 \pi i} \int_{\gamma-i \infty \infty}^{\gamma+i \infty}\left[A_{n} e^{-R_{n} z}+B_{n} e^{R_{n} z}+\frac{Q}{2 R_{n} K_{n}}\right. \\
& \left.\times\left(e^{-\left(R_{n}\left(z-H_{S}\right)+t_{0} s\right)}-e^{R_{n}\left(z+H_{S}\right)-t_{0} s}\right) \cdot H\left(z-H_{s}\right)\right] e^{s t} \mathrm{~d} s
\end{aligned}
$$

for $n=1, \cdots, N-1$, where $R_{n}=\sqrt{s / K_{z_{n}}}, H_{s}$ is the height of the source (located in $x=0$ ) and $\mathrm{H}$ is the Heaviside function that multiplies the part that is valid only in the sub-layer that contains the source, $A_{n}$ and $B_{n}$ $(n=1, \cdots, N-1)$ are the integration constants, they are determinate by solving the linear system resulting from the application of the boundary and interfaces conditions.

More details on the ADMM approach can be found on [8] and [11].

Finally, we would like to point out that since we succeed in the task of searching solution for the puff problem (1), we are confident to affirm that we pave the road to mitigate the previous Gaussian assumptions in the $x$ and $y$ directions, working out the three-dimensional advection-diffusion equation with puff source by the ADMM approach.

Once the solution for single puff is know, we claim that the puff solution reads like the summation of the contribution of all puffs emitted by the source. This procedure leads to the solution:

$$
\begin{aligned}
& c_{n_{\text {puff }}}(z, t) \\
& =\sum_{p=1}^{P} \Delta M_{p}\left\{\int_{t=0}^{\infty} c_{n}(z, t) H\left(t-t_{0_{p}}\right) \mathrm{d} t\right\}
\end{aligned}
$$

for $p=1: P$, where $P$ is the total number of puffs emitted, $\Delta M_{p}$ is the mass carried out for the $p^{\text {th }}$ puff and $c_{n_{p u f f}}$ is the one-dimensional puff solution. To obtain the searched three-dimensional solution, we make the assumption that the solution in the $x$ and $y$ directions are described by Gaussian solution. Henceforth, the threedimensional puff solution, assuming point source at $x=y=0$, has the form:

$$
c_{\text {puff }}(x, y, z, t)=c_{n_{\text {puff }}} \psi_{\text {puff }}(x, y, t),
$$

noticing that the function $\psi_{p u f f}$ is given by Equation (4). Here $C_{p u f f}(x, y, z, t)$ is the searched puff solution. Further, $x_{0}$ and $y_{0}$ are the puff centroid coordinates expressed in terms of $x$ and $y$ wind component as:

$$
\begin{aligned}
& x=u \Delta t, \\
& y=v \Delta t,
\end{aligned}
$$

where $u$ and $v$ are respective the wind components in the $x$ and $y$ directions. Here $\Delta t$ is the time interval of the puff emission. In addition, we must recall that $\sigma_{x}$ and $\sigma_{y}$ are the standard deviation for $x$ and $y$ directions [12].

\section{Experimental Data and Vertical Turbulence Parameterization}

The performances of the present models were evaluated against experimental data of Copenhagen $[12,15]$ data set. 
In the Copenhagen experiment the tracer SF6 was released without buoyancy from a tower at a height of $115 \mathrm{~m}$, and collected at the ground-level positions at a maximum of three crosswind arcs of tracer sampling units. The sampling units were positioned, at the ground level, 2-6 km from the point of release. The site was mainly residential with a roughness length of $0.6 \mathrm{~m}$. The meteorological conditions during the dispersion experiments ranged from moderately unstable to convective. We used the values of the crosswind-integrated concentrations normalized with the tracer release rate from [12]. Generally, the distributed data set contains hourly mean values of concentrations and meteorological data. However, in this model validation, we used data with a greater time resolution kindly made available by Gryning and described in [3]. In particular, we used 20 minutes averaged measured concentrations and 10 minutes averaged values for meteorological data Tables 1, 2 and $\mathbf{3}$ report the friction velocity, the Monin-Obukhov length and boundary layer height (only one value for each run), respectively, used in the simulations. The puffs considered here are emitted in time intervals $\Delta t_{1}=120 \mathrm{~s}$. and the calculation of the concentration of pollutants is made with a time resolution $\Delta t_{2}=30 \mathrm{~s}$.

In order to evaluate the performance of the puff models against experimental ground-level concentration. we have to introduce a boundary layer parameterization.

The $K$ theory assumes that concentration turbulent fluxes are proportional to the mean concentration gradient. The reliability of the $K$-approach strongly depends on the way the eddy diffusivity is determined on the basis of the turbulence structure of the ABL, and on the model's ability to reproduce experimental diffusion data.

Table 1. The friction velocity $(\mathrm{m} / \mathrm{s})$ for the time $t=n \Delta t$ for $n=1: 12$ and $\Delta t=10$ minutes.

\begin{tabular}{ccccccccc}
\hline Run & 1 & 2 & 3 & 4 & 5 & 7 & 8 & 9 \\
\hline 1 & 0.36 & 0.68 & 0.46 & 0.56 & 0.58 & 0.48 & 0.65 & 0.72 \\
2 & 0.37 & 0.67 & 0.45 & 0.51 & 0.52 & 0.48 & 0.79 & 0.73 \\
3 & 0.40 & 0.81 & 0.47 & 0.37 & 0.51 & 0.57 & 0.67 & 0.60 \\
4 & 0.43 & 0.68 & 0.39 & 0.44 & 0.58 & 0.62 & 0.67 & 0.59 \\
5 & 0.35 & 0.75 & 0.39 & 0.48 & 0.59 & 0.53 & 0.68 & 0.65 \\
6 & 0.34 & 0.74 & 0.40 & 0.48 & 0.52 & 0.65 & 0.65 & 0.71 \\
7 & 0.42 & 0.76 & 0.40 & 0.39 & 0.52 & 0.63 & 0.68 & 0.73 \\
8 & 0.43 & 0.82 & 0.41 & 0.40 & 0.45 & 0.65 & 0.67 & 0.73 \\
9 & 0.40 & 0.76 & 0.31 & 0.39 & 0.44 & 0.66 & 0.73 & 0.73 \\
10 & 0.37 & 0.73 & 0.34 & 0.39 & 0.44 & 0.62 & 0.73 & 0.66 \\
11 & 0.35 & 0.69 & 0.39 & 0.39 & 0.44 & 0.52 & 0.75 & 0.67 \\
12 & 0.36 & 0.66 & 0.40 & 0.39 & 0.43 & 0.62 & 0.69 & 0.74 \\
\hline
\end{tabular}

Table 2. The Monin-Obukhov length (m) for the time $t=n \Delta t$ for $n=1: 12$ and $\Delta t=10$ minutes.

\begin{tabular}{ccccccccc}
\hline$N^{\text {Run }}$ & 1 & 2 & 3 & 4 & 5 & 7 & 8 & 9 \\
\hline 1 & -26 & -178 & -152 & -75 & -492 & -71 & -71 & -793 \\
2 & -23 & -227 & -194 & -42 & -215 & -80 & -85 & -471 \\
3 & -83 & -311 & -106 & -23 & -368 & -64 & -47 & -202 \\
4 & -42 & -160 & -101 & -32 & -735 & -111 & -49 & -366 \\
5 & -36 & -203 & -129 & -71 & -366 & -177 & -45 & -633 \\
6 & -42 & -286 & -70 & -80 & -273 & -67 & -63 & -13588 \\
7 & -47 & -155 & -83 & -83 & -273 & -87 & -41 & -593 \\
8 & -38 & -228 & -60 & -101 & -262 & -71 & -47 & -471 \\
9 & -83 & -184 & -106 & -129 & -395 & -56 & -70 & -389 \\
10 & -21 & -389 & -42 & -129 & -395 & -111 & -64 & -375 \\
11 & -32 & -133 & -101 & -129 & -395 & -215 & -52 & -262 \\
12 & -29 & -375 & -70 & -129 & -759 & -123 & -39 & -252 \\
\hline
\end{tabular}

Table 3. Boundary layer height for the nine experiments (hourly data).

\begin{tabular}{ccccccccc}
\hline Run & 1 & 2 & 3 & 4 & 5 & 7 & 8 & 9 \\
\hline $\boldsymbol{h}$ & 1980 & 1920 & 1120 & 390 & 820 & 1850 & 810 & 2090 \\
\hline
\end{tabular}

We are aware of the great variety of parameterization of the eddy-diffusivity coefficient inherent to the $K$-model [10]. Most of them are based on similarity theory, and give different results for the same atmospheric stability, as well as discontinuities and jumps at the transition between different stability regimes of the ABL.

However, in this work, we select two formulations for the eddy diffusivity coefficient for the vertical dispersion. The first one is discussed by Pleim and Chang [16] and written as:

$$
K(z)=k w_{*} z\left(1-\frac{z}{h}\right)
$$

The second one, is the eddy diffusivity suggested by Degrazia et al. [17] which reads like:

$$
K(z)=\frac{0.55}{4} \frac{\sigma_{w} z}{\left(f_{m}^{*}\right)_{w}},
$$

where the vertical speed variance $\sigma_{w}$ is defined as:

$$
\sigma_{w}^{2}=1.06 c_{w} \frac{\psi^{2 / 3}}{\left(f_{m}^{*}\right)_{w}^{2 / 3}}\left(\frac{z}{h}\right)^{2 / 3} w_{*}^{2}
$$

where $c_{w}=0.36, w_{*}$ is the convective velocity scale, $\left(f_{m}^{*}\right)_{w}$ is the non-dimensional frequency of the vertical spectral peak expressed by following form: 


$$
\left(f_{m}^{*}\right)_{w}=\frac{z}{\left(\lambda_{m}\right)_{w}}
$$

and $\psi$

$$
\psi=1.5-1.2\left[\left(\frac{z}{h}\right)^{1 / 3}\right]
$$

where $\left(\lambda_{m}\right)_{w}$ is the wave associated length with the maximum vertical specter given by:

$$
\left(\lambda_{m}\right)_{w}=\left\{\begin{array}{lc}
\frac{z}{0.55-0.38\left|\frac{z}{L}\right|} \quad 0 \leq z \leq|L| \\
5.9 z & |L| \leq z \leq 0.1 h s \\
1.8 h\left[1-\exp \left(\frac{-4 z}{h}\right)-0.0003 \exp \left(\frac{8 z}{h}\right)\right] & 0.1 h<z
\end{array}\right.
$$

where $L$ is the Monin-Obukov length.

The motivation for our choice is grounded in the fairly good results reported in $[3,18,19]$, for these parameterizations. This justification is reinforced by the comparative study of eddy-diffusivity parameterizations appearing in [20,21].

\section{Results}

We have applied the model using the Copenhagen experimental datasets [15].

The validation has to be considered preliminary one. As a matter of fact, we have checked only two boundary layer parameterization with data referring to continuous emission in variable meteorology (with time resolution of 10 minutes) and in receptors points far from the source (2 - $6 \mathrm{~km}$ ). In Figure 1 the scatter diagram of the results of the two models using the Plaim and Chang eddy diffusivity profile is shown. While in Figure 2 the scatter diagram with the Degrazia et al. profile is presented.

In Table 4 some well-known statistical indices of models performances are reported. They are suggested and discussed in [22-23] and defined in the following way:

$$
\text { nmse }=\frac{\overline{\left(C_{o}-C_{p}\right)^{2}}}{\overline{C_{o} \overline{C_{p}}}}: \quad \text { normalized mean square error }
$$

$r=\frac{\overline{\left(C_{o}-\overline{C_{o}}\right)\left(C_{p}-\overline{C_{p}}\right)}}{\sigma_{o} \sigma_{p}}$ correlation coefficient

fa2 = data for which: fraction of data $(\%$, normalized $0.5 \leq C_{p} / C_{o} \leq 2: \quad$ to 1$)$

$$
\mathrm{fb}=2 \frac{\overline{C_{o}}-\overline{C_{p}}}{\overline{C_{o}}+\overline{C_{p}}}: \quad \text { fractional bias }
$$

$$
\mathrm{fs}=2 \frac{\sigma_{o}-\sigma_{p}}{\sigma_{o}+\sigma_{p}}: \quad \text { fractional standard deviations }
$$

where subscripts $\boldsymbol{o}$ and $\boldsymbol{p}$ refer to observed and predicted quantities, respectively, $\sigma$ is the standard deviation, $\boldsymbol{C}$ the concentration and the over bar indicates an averaged value. The statistical index fb says if the predicted quantities underestimate or overestimate the observed ones. fa2 is the fraction of $\boldsymbol{C}_{\boldsymbol{p}}$ values (normalized to 1) within a factor two of corresponding $\boldsymbol{C}_{\boldsymbol{o}}$ values. The statistical index nmse represents the model values dispersion in respect to data dispersion. The best results are expected to have values near zero for the indices nmse, fb and fs, and near one in the indices $\mathbf{r}$ and $\mathbf{f a} 2$.

In Table 4 are reported the results of the statistical indices of the puff models using ADMM and GILTT, respectively, and considering the eddy diffusivity suggested by Pleim Chang [16] and Degrazia et al. [17], respectively.

The analysis of the statistical evaluation shows a reasonable agreement between the computed values against the experimental ones, without any significant difference between the two models and the two parametrization of vertical turbulence.

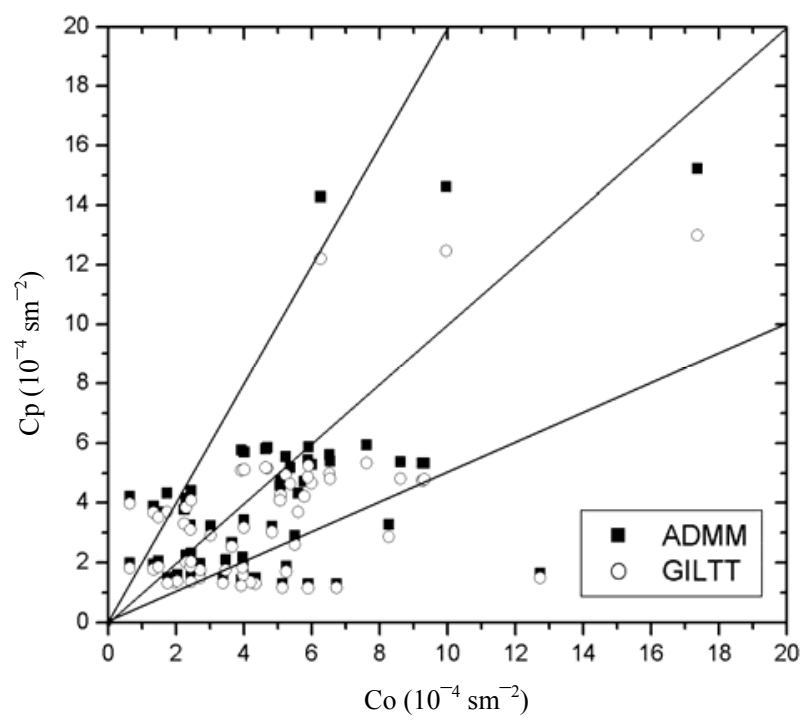

Figure 1. Scatter plotted of observed (Co) and computed (Cp) crosswind ground-level integrated concentration, normalised with emission ( $c / Q)$, with eddy diffusivities from Pleim Chang. The data between two external lines are in a factor of 2 . 


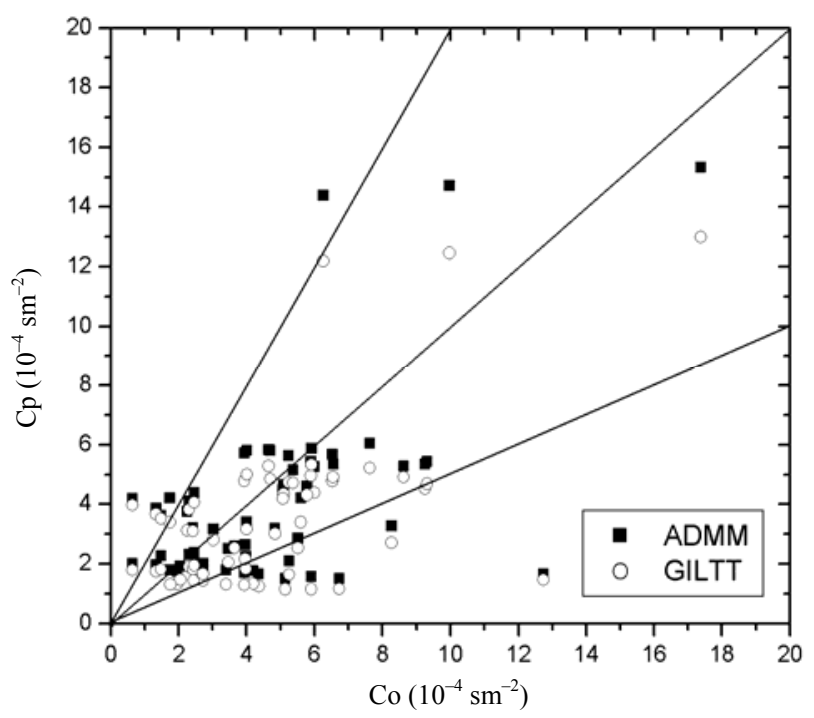

Figure 2. Scatter plotted of observed (Co) and computed (Cp) crosswind ground-level integrated concentration, normalised with emission (c/Q), with eddy diffusivities from Degrazia. The data between two external lines are in a factor of 2.

Table 4. Statistical evaluation of model results.

\begin{tabular}{ccccccc}
\hline Model & $\begin{array}{c}\text { Edyy } \\
\text { diffusivity }\end{array}$ & nmse & r & fa2 & fb & fs \\
\hline ADMM & Eq. (41) & 0.42 & 0.58 & 0.72 & 0.16 & 0.01 \\
ADMM & Eq. (42) & 0.40 & 0.58 & 0.75 & 0.14 & 0.02 \\
GILTT & Eq. (41) & 0.48 & 0.57 & 0.70 & 0.28 & 0.16 \\
GILTT & Eq. (42) & 0.48 & 0.57 & 0.72 & 0.29 & 0.17 \\
\hline
\end{tabular}

\section{Conclusions}

We presented a puff model where the vertical concentration distribution is not Gaussian but is describe by two new solutions of the advection-diffusion equation that accept eddy diffusion coefficient with any restriction in their height function.

As preliminary evaluation of the model performances, the results predicted by the puff model, using two different turbulence parameterizations where compared with data from the well-known Copenhagen data set, but with a greater time resolution respect to the original one.

Analysis of the results obtained and the application of well-known statistical indices show that both approaches considered produce a good fit for the ground level concentration data. There are not significant differences between the two solutions and the two eddy diffusivity parameterizations in the range of experimental data.
The preliminary results confirm the applicability of the approach proposed and are promising for future work: evaluation of other eddy diffusivity parameterizations and using the ADMM and GILTT solutions in the lateral dispersion also.

\section{Acknowledgements}

The authors are gratefully indebted to CNPq, FAPERGS, CNR and ENVIREN for the partial financial support of this work.

\section{REFERENCES}

[1] R. Lupini and T. Tirabassi, "Solution of the AdvectionDiffusion Equation by the Moments Method," Atmospheric Environment, Vol. 17, No. 5, 1983, pp. 965-971. doi:10.1016/0004-6981(83)90248-2

[2] T. Tirabassi and U. Rizza, "A Practical Model for the Dispersion of Skewed Puffs," Journal of Applied Meteorology, Vol. 34. No. 4, 1995, pp. 989-99. doi:10.1175/1520-0450(1995)034<0989:APMFTD $>2.0 . C$ $\mathrm{O} ; 2$

[3] T. Tirabassi and U. Rizza, "Boundary Layer Parameterization for a Non-Gaussian Puff Model," Journal of Applied Meteorology, Vol. 36, No. 8, 1997a, pp. 1031-1037. doi:10.1175/1520-0450(1997)036<1031:BLPFAN $>2.0 . C$ $\underline{\mathrm{O} ; 2}$

[4] T. Tirabassi and U. Rizza, "A Non-Gaussian Puff Model," Nuovo Cimento, Vol. 20C, 1997b, pp. 453-460.

[5] S. Wortmann, M. T. M. Vilhena, D. D. Moreira and D. Buske, "A New Analytical Approach to Simulate the Pollutant in the PBL," Atmospheric Environment, Vol. 39, No. 34, 2005, pp. 2171-2178.

doi:10.1016/j.atmosenv.2005.01.003

[6] D. M. Moreira, M. T. Vilhena, D. Buske and T. Tirabassi, "The GILTT Solution of the Advection-Diffusion Equa tion for an Inhomogeneous and Nonstationary PBL," Atmospheric Environment, Vol. 40, No. 17, 2006, pp. 31863194. doi:10.1016/j.atmosenv.2006.01.035

[7] M. T. Vilhena, U. Rizza, G, Degrazia, C. Mangia, D. M. Moreira and T. Tirabassi, "An Analytical Air Pollution Model: Development and Rvalution," Contribution Atmospheric Physics, Vol. 71, 1998, pp. 818-827.

[8] D. M. Moreira, M. T. Vilhena, M. T. T. Tirabassi, C. P Costa and B. Bodmann, "Simulation of Pollutant Dispersion in the Atmosphere by the Laplace Transform: The ADMM Approach," Water, Air and Soil Pollution, Vol. 177, No. 1-4, 2006a, pp. 411-439. doi:10.1007/s11270-006-9182-2

[9] P. Zannetti, “Air Pollution Modeling," Computer Technology Publications, Southampton, UK, 1990.

[10] J. H. Seinfeld and S. N. Pandis, "Atmospheric Chemistry and Physics," John Wiley \& Sons, New York, 1998, pp. 1326.

[11] C. P. Costa, M. T. Vilhena, D. M. Moreira and T. Tira- 
bassi, "Semi-Analytical Solution of the Steady ThreeDimensional Advection-Diffusion Equation in the Planetari Boundary Layer," Atmospheric Environment, Vol. 40, 2006, pp. 5659-5669.

doi:10.1016/j.atmosenv.2006.04.054

[12] S. E. Gryning, A. A. M. Holtslag, J. S. Irwin and B. Siversten, "Applied Dispersion Modelling Based on Meteorological Scaling Parameters," Atmospheric Environment, Vol. 21, 1987, pp. 79-89. doi:10.1016/0004-6981(87)90273-3

[13] C. F. Segatto and M. T. M. B. Vilhena, "The Stateof-the-Art of the LTSN Method," Proceeding of M\&C'99 Conference on Mathematics and Computation, Reactor Physics and Environmental Analysis in Nuclear Applications, Vol. 3, No. 2, 1999, pp. 1618-1631.

[14] D. M. Moreira, M. T. Vilhena, D. Buske and T. Tirabassi, "The State-of-Art of the GLTT Method to Simulate Pollutant Dispersion in the Atmosphere," Atmospheric Research, Vol. 92, 2009, pp. 1-17. doi:10.1016/j.atmosres.2008.07.004

[15] S. E. Gryning and E. Lyck, "Atmospheric Dispersion from Elevated Sources in an Urban Area: Comparison between Tracer Experiments and Model Calculations," Journal of Climate Applied Meteorology, Vol. 23, No. 4, 1984, pp. 651-660. doi:10.1175/1520-0450(1984)023<0651:ADFESI >2.0.C $\underline{\mathrm{O} ; 2}$

[16] J. Pleim and J. S. Chang, "A Non-Local Closure Model for Vertical Mixing in the Convective Boundary Layer," Atmospheric Environment, Vol. 26, No. 6, 1992, pp. 965981.

[17] G. A. Degrazia, D. M. Moreira and M. T. M. B. Vilhena, "Derivation of an Eddy Diffusivity Depending on Source
Distance for Vertically in Homogeneous Turbulence in a Convective Boundary Layer," Journal of Applied Meteorology, 2001, pp. 1233-1240. doi:10.1175/1520-0450(2001)040<1233:DOAEDD $>2.0$. $\underline{\mathrm{CO} ; 2}$

[18] C. Mangia, D. M. Moreira I. Schipa G. A. Degrazia, T. T. Tirabassi and U. Rizza. "Evaluation of a New Eddy Diffusivity Parameterisation from Turbulent Eulerian Spectra in Different Stability Conditions," Atmospheric Environment, Vol. 36, No. 1, 2002, pp. 67-76. doi:10.1016/S1352-2310(01)00469-1

[19] D. M. Moreira, J. C. Carvalho, A. G. Goulart and T. Tirabassi, "Simulation of the Dispersion of Pollutants Using Two Approaches for the Case of a Low Source in a SBL: Evaluation of Turbulence Parameterisations," Water Air and Soil Pollution, Vol. 161, No. 1-4, 2005, pp. 285-297. doi:10.1007/s11270-005-4287-6

[20] T. Tirabassi, F. Baffo and D. M. Moreira, "Eddy Diffusivity Parameterizations and Improve Features in an Analytical Multilayer Dispersion Model," Proceeding of XIII Brazilian Congress of Meteorology, Fortaleza (Br), August 2004.

[21] D. Buske, M. T. Vilhena, D. M. Moreira and T. Tirabassi, "Evaluation of Eddy Diffusivity Parameterisations by an Analytical Solution of the Advection-Diffusion Equation," Proceeding of XIV Brazilian Congress of Meteorology, Florianopolis (Br), November 2006.

[22] S. R. Hanna, "Confidence Limit for Air Quality Models as Estimated by Bootstrap and Jackknife Resampling Methods," Atmospheric Environment, Vol. 23, No. 6, 1989, pp. 1385-1395. doi:10.1016/0004-6981(89)90161-3

[23] J. C. Chang and S. R. Hanna. "Air Quality Model Performance Evaluation," Meteorological Atmosphere Physics, Vol. 87, No. 1-3, 2004, pp. 167-196. 